\title{
Far-Infrared Blocked Impurity Band Detector Development
}

\author{
H. H. Hogue, M.T. Guptill, J. C. Monson, and J. W. Stewart \\ DRS Sensors \& Targeting Systems, Cypress, CA \\ J. E. Huffman \\ Lawrence Semiconductor Research Laboratory, Tempe, AZ \\ M G Mlynczak and M N Abedin \\ NASA Langley Research Center, Hampton, VA
}

\begin{abstract}
DRS Sensors \& Targeting Systems, supported by detector materials supplier Lawrence Semiconductor Research Laboratory, is developing far-infrared detectors jointly with NASA Langley under the Far-IR Detector Technology Advancement Partnership (FIDTAP). The detectors are intended for spectral characterization of the Earth's energy budget from space. During the first year of this effort we have designed, fabricated, and evaluated pilot Blocked Impurity Band (BIB) detectors in both silicon and germanium, utilizing pre-existing customized detector materials and photolithographic masks. A second-year effort has prepared improved silicon materials, fabricated custom photolithographic masks for detector process, and begun detector processing. We report the characterization results from the pilot detectors and other progress.
\end{abstract}

Keywords: far-infrared detectors, silicon, germanium, blocked impurity band, space based, earth observing

\section{INTRODUCTION}

Measuring the energy absorbed and reemitted to space is a long standing problem that has taken on increased urgency today with the realization that human activities are impacting the Earth's energy balance. Since the 1960's integral energy flow measurements have been performed from space with increasing spatial and temporal coverage, but only recently have satellite based instruments begun to obtain important spectral measurements. The Atmospheric Infrared Sounder (AIRS) and the Moderate Resolution Imaging Spectrometer (MOSIS) instruments on the EOS-Aqua satellite and the soon to be flown Infrared Atmospheric Sounding Interferometer (IASI) on a METOP European polar-orbiting satellites and Crosstrack Infrared Sounder (CrIS) instrument on the NPOESS satellites have spectral capability out to only $15 \mu \mathrm{m}$ in the infrared, limited by the availability of mature far-infrared detectors. The background, status, and plans for extended wavelength measurements were discussed in an earlier conference paper. ${ }^{1}$

Full characterization of the Earth's emission spectrum from space will require measurements out to about $100 \mu \mathrm{m}$ in wavelength. ${ }^{2}$ NASA Langley Research Center has taken a leading role in developing instrumentation to pursue these longer wavelength measurements. A stepping stone has been the development of the Far-Infrared Spectroscopy of the Troposphere (FIRST) instrument ${ }^{3}$ for balloon-borne measurements to $100 \mu \mathrm{m}$ in the far-infrared. The FIRST instrument uses a silicon bolometer cooled to $4 \mathrm{~K}$ by liquid helium cryogen. Application of FIRST-type studies to satellites is hindered by this detector technology, because bolometers have relatively low efficiency compared to photon detectors, and at their low operating temperature long-term cooling is currently not practical.

A further step toward space implementation of a FIRST-type instrument is the partnership developed between NASA Langley and DRS Sensors \& Targeting Systems to develop high efficiency far-infrared photon detectors capable of operating at higher temperatures than those currently in FIRST. At operating temperature of at least $10 \mathrm{~K}$, mechanical cryocoolers are in development that will enable long-term space based missions. This partnership, the Far-Infrared Detector Technology Advancement Partnership (FIDTAP) ${ }^{1}$, began detector development efforts about one year ago as an equal cost share between the parties. The first development cycle has been completed and a second cycle is in progress. This paper reports technical progress and plans. 


\section{OBJECTIVE AND APPROACH}

The main requirements of photon detectors for full Earth spectrum, far-infrared spectral measurements from space are listed in Table I. At the outset of the current development effort, two technological approaches to these detector objectives were defined. Both were implementations of Blocked Impurity Band (BIB) detectors, ${ }^{4}$ based either on silicon or germanium. Silicon based BIB detectors, doped with arsenic (Si:As) or antimony (Si:Sb), are in common usage for high quantum efficiency detection for the 5 to $40 \mu \mathrm{m}$ wavelength range. Arrays of these detectors at formats up to $256 \mathrm{x}$ 256, mated to silicon readout multiplexers, are currently in the instruments aboard the Spitzer Space Telescope, and they are widely used as focal plane imagers at high-altitude ground-based observatories. Megapixel (1024 x 1024) BIB detector arrays for low-background space applications have been developed ${ }^{5}$ for the Wide-field Infrared Survey Explorer (WISE) mission, for launch in late 2009, and for the MIRI instrument for the James Webb Space Telescope, to launch several years later. Megapixel BIB focal plane arrays for higher-flux operation are under development for ground-based instruments. ${ }^{6}$ While current silicon BIB detectors are limited to $40 \mu \mathrm{m}$, this wavelength cutoff depends on the dopant concentration in the main infrared sensitive layer of the detector structure. The extension of silicon BIB detector wavelength range to at least $70 \mu \mathrm{m}$ appears to be feasible by increasing doping level to several times the levels typically used. Wavelength response to $70 \mu \mathrm{m}$ has been reported for a different type of highly doped silicon device. ${ }^{7}$

Table I. Detector Specifications

\begin{tabular}{|c|c|}
\hline Parameter & Value \\
\hline Operating temperature & At least $10 \mathrm{~K}$ \\
Specific detectivity, D* & $>10^{10} \mathrm{~cm} \mathrm{~Hz}^{1 / 2} / \mathrm{W}$ \\
Nominal scene temperature & $230 \mathrm{~K}$ \\
Frequency response & $>1 \mathrm{kHz}$ \\
Detector size & $<200 \mu \mathrm{m}$ diameter \\
Detector format & Single element; demonstrated \\
& path to arrays \\
Continuous wavelength coverage & 10 to $100 \mu \mathrm{m}$ \\
\hline
\end{tabular}

Germanium based BIB detectors are another option which has been demonstrated ${ }^{8}$. The shallower dopant energy level in germanium allows BIB detectors from this material to be sensitive to wavelengths of $200 \mu \mathrm{m}$ or more, at doping levels lower than utilized for silicon BIB detectors. With proper design it may be feasible to operate BIB germanium detectors at sufficiently high temperature to meet the requirements of Table I. However, germanium BIB detectors have not yet achieved sufficient technological readiness for science instrumentation. The main limitation has been obtaining sufficiently good characterization of as-grown doped and undoped germanium layers that growth processes can be adapted to achieve the desired layer make-up. Germanium detector processing is a secondary limitation. Approaches to provide reliable metal to semiconductor contacts, insulating layers, and surface passivation are not as well developed for germanium as for silicon processing. Photoconductive germanium detectors, which are simpler in structure than BIB detectors and operate at temperature below $4 \mathrm{~K}$, have continued to be used for far-infrared detection in missions such as Spitzer.

At the initiation of the DRS/Langley detector development project, DRS had available preliminary BIB detector structures in both highly doped silicon and germanium, resulting from earlier research and development activities. It was decided to utilize these materials to process and evaluate pilot detectors to narrow the approach to be implemented in a subsequent phase of detector development aimed at the specific objectives of Table I. 


\section{FABRICATION OF PILOT DETECTORS}

The starting material for silicon or germanium BIB detectors consists of layers of single crystal silicon or germanium, with the principal detector layer being doped with a Group III or Group IV species to make it responsive to infrared wavelengths. Table II lists the relevant material parameters of the infrared active layers for the starting material for the pilot lot. The arsenic-doped silicon material ranged from about 1.5 to about 4 times the dopant concentration typically employed in BIB detectors with cut-off wavelengths of about $28 \mu \mathrm{m}$.

Table II. Starting BIB Detector Material Parameters

\begin{tabular}{|c|c|c|}
\hline Material & Active Layer Doping & $\begin{array}{c}\text { Active Layer } \\
\text { Thickness }\end{array}$ \\
\hline $\mathrm{Si}: \mathrm{As}$ & $1.4 \times 10^{18} \mathrm{~cm}^{-3}$ & $10 \mu \mathrm{m}$ \\
\hline $\mathrm{Si}: \mathrm{As}$ & $3 \times 10^{18} \mathrm{~cm}^{-3}$ & $10 \mu \mathrm{m}$ \\
\hline $\mathrm{Ge}: \mathrm{B}$ & $3 \times 10^{16} \mathrm{~cm}^{-3}$ & $10 \mu \mathrm{m}$ \\
\hline
\end{tabular}

Processing detectors from these materials involved patterned silicon or germanium etches, patterned ion implantations (followed by furnace annealing to activate implants), deposition and patterning of silicon oxides and metals, and other steps. A photolithographic mask set is needed to create the photoresist structures for detector patterning of active detection areas, electrical traces, and other features. For the pilot lot we used an existing mask set that would provide wirebondable single circular detector elements with diameters of $500 \mu \mathrm{m}$ and $1000 \mu \mathrm{m}$. Although these detector sizes are larger than that desired in the final detector design, the use of existing masks for the pilot lot offered some savings. Results for smaller detector sizes can be reliably inferred from measurements of larger detectors, because conventional BIB detector dark and photocurrents scale accurately with area.

The fabrication of both the silicon and germanium pilot detector types was completed and wafers with the BIB detector and representative wafers were diced into chips of suitable size for test packaging. Each chip contained several detectors of each of the two detector sizes on the mask set. Chips were packaged for test by attaching them with epoxy to ceramic leadless chip carriers (LCC) and wirebonding selected individual detectors to LCC electrical pads. Figure 1 shows the test packages prepared in this way.

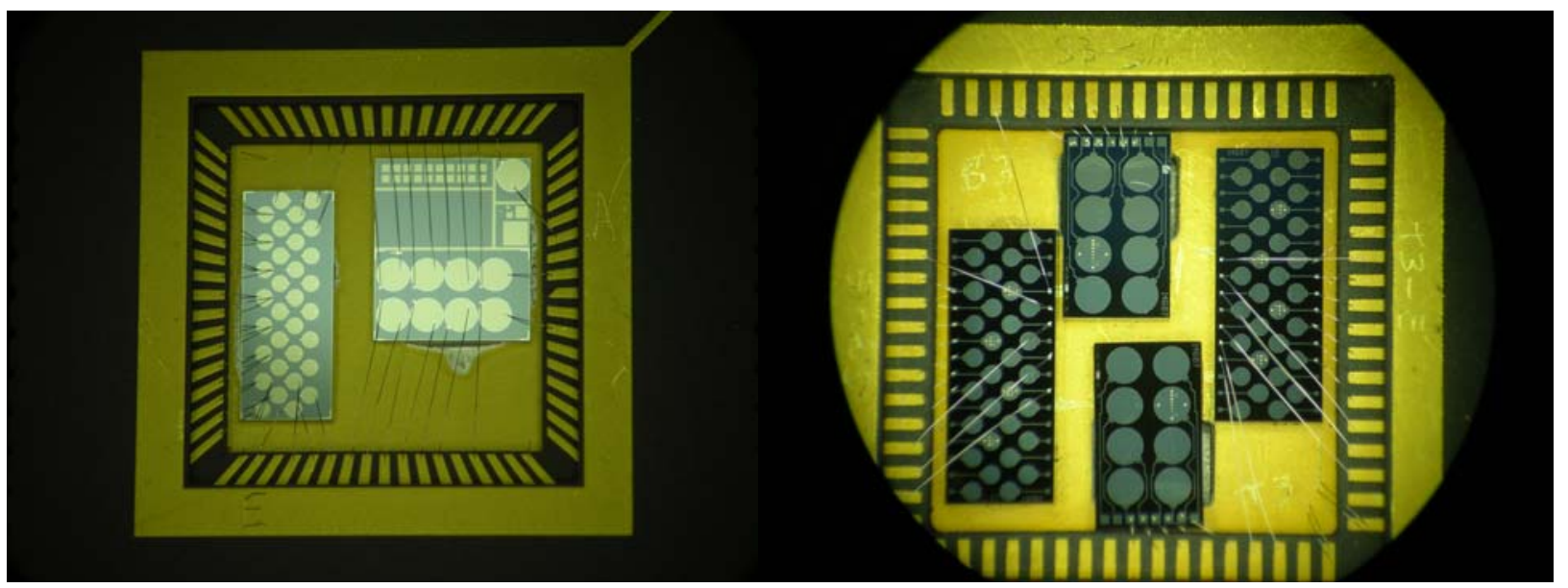

Figure 1. Microscope photographs of central portion of germanium BIB detector test package (left) and silicon BIB detector test package (right). The silicon test package included detectors of both dopant levels and both sizes 


\section{TESTING OF PILOT DETECTORS}

DRS has the capability to test BIB detector devices at all scales of integration, from individual detectors to large multiplexed detector arrays to modules that integrate detector arrays with drive and data acquisition electronics. The principle item of test equipment required for BIB device testing is a cryostat of helium-flow type (helitran) or heliumreservoir type (dewar). For testing the current pilot detectors we used an existing liquid helium dewar with a liquidnitrogen temperature intermediate cold shield. The dewar base, which includes the cryogen chambers and vacuum enclosure with necessary pumping and cryogen filling ports, had been obtained from Janis Research and configured with a custom temperature controlled stage and socket for operating LCC-packaged detectors. Operating temperature may be controlled at any value between $\sim 5 \mathrm{~K}$ and $\sim 20 \mathrm{~K}$. The DRS configuration of the test dewar had also included a liquidhelium temperature inner radiation shroud, liquid-nitrogen temperature intermediate cold shroud, and vacuum shroud. These features are illustrated in Figure 2. The vacuum housing and radiation shields are optically ported to allow infrared radiation to reach test device from an external source. A gravity operated cold shutter on the liquid-helium shield can be closed to provide dark conditions for dark current testing. For our testing at far-infrared wavelengths, removable filters on the liquid-nitrogen and liquid-helium temperature shrouds were not installed, and the KRS-5 optical window was replaced with a silicon window.

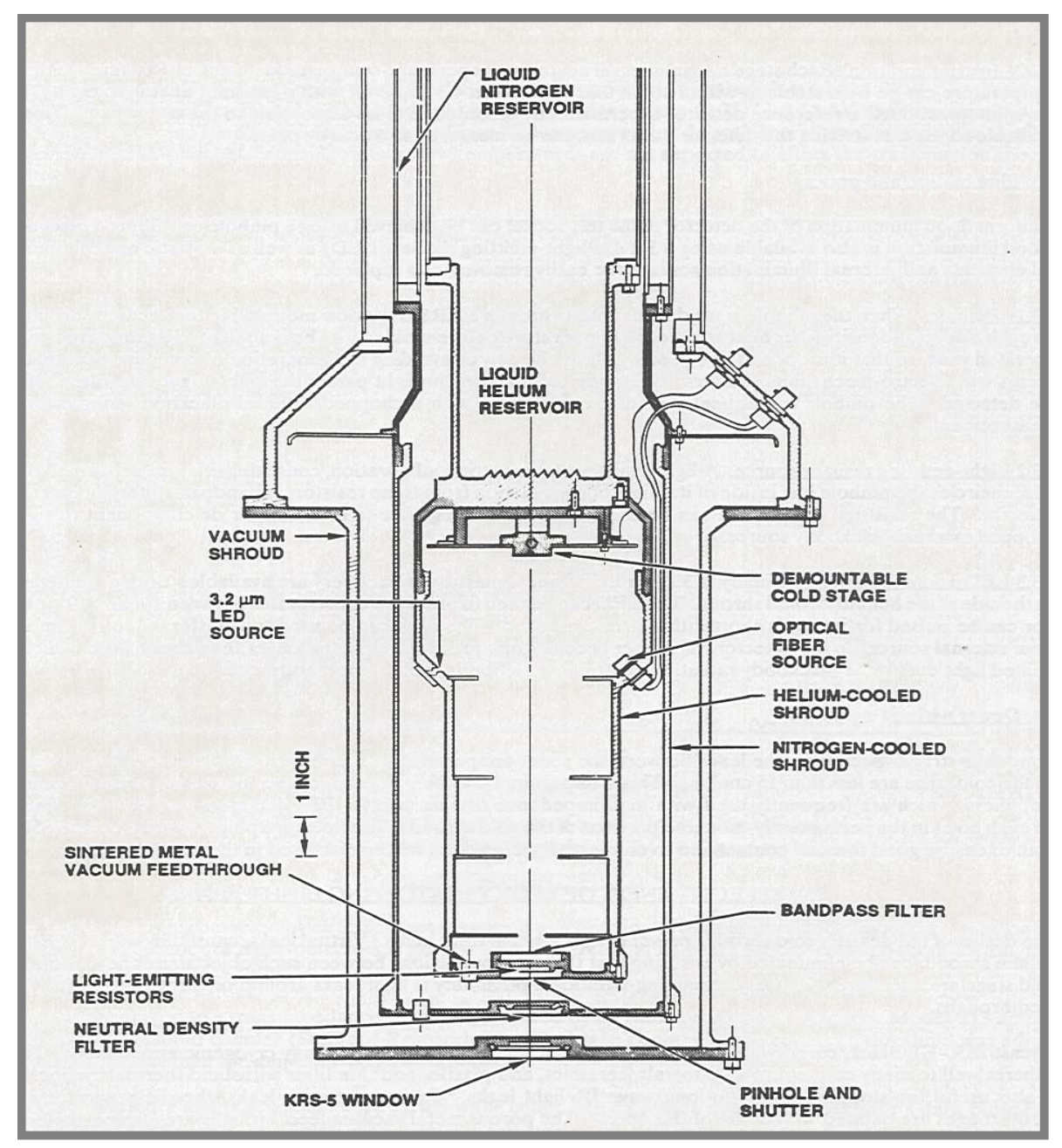

Figure 2. Test dewar illustration 
The test detectors were evaluated by directly measuring their output current in response to a bias voltage, which was slowly swept from zero volts to full operating bias (dependent on detector type). These bias sweeps, also called I-V curves, were taken under dark and illuminated conditions for a range of operating temperatures and for different spectral content of the externally applied illumination. A Keithley Instruments Model 4200 Semiconductor Characterization System was used to apply the bias voltage and capture the current data into Excel spreadsheets for analysis.

Far-infrared illumination was provided a blackbody source operated at $500 \mathrm{~K}$ temperature and filtered through a longpass infrared filter. A filter set for this purpose was obtained from Lakeshore and included filters at cut-on wavelengths of 30, 50, 70, and 114 microns. These are scatter-type filters that do not have a sharp cut-on wavelength; however, they provided illumination sources with a sufficiently different spectral contents and allowed estimates of detector spectral performance to be made by comparing detector outputs for the different filters from the set. Spectral testing is discussed below.

\subsection{Germanium detector basic operability}

The germanium detectors are doped with boron, a p-type dopant. In typical BIB detector operating mode they would have negative voltage applied to the top contact relative to the substrate, to deplete the active layer of charge carriers. However, the germanium detectors were found to operate only in opposite polarity. This anomalous operation is not well understood, but two factors may be involved. 1) The make-up of the germanium blocking layer. As discussed on page 2, attempts to develop germanium detectors have been limited by knowledge of the unintentional doping present in the as-deposited germanium layers. The blocking layer, the undoped detector layer in the BIB detector structure that blocks leakage current and allows higher temperature operation, may be particularly impacted by the presence of unintended atomic species. In normal polarity, large voltage may be needed to overcome the screening effect of impurities in the blocking layer to achieve effective depletion into the underlying active layer. 2) It is likely that the electrical contacts to the germanium detectors were not doped to the metal-transition level and are quite not-ohmic at operating temperature. Indeed, the operation of the devices in opposite polarity (accumulation mode) indicates the presence of a non-ohmic electrical contact, since current did not flow until a bias voltage of about $0.7 \mathrm{~V}$ has been applied, whereas BIB detectors with ohmic contacts turn on rapidly with applied bias.

\subsection{Germanium detector dark current performance}

Figure 3 plots representative dark I-V curves for the two sizes of detectors in the test germanium detector test package. In order to determine whether these devices are functioning as BIB detectors, the data for $1.5 \mathrm{~V}$ bias were plotted vs the reciprocal of the temperature. This resulted in a thermally activated behavior of the dark current, as shown in Figure 4. The data for both small and large detectors were fit with an exponential form over the region where the current values are sufficiently large to be accurately measured and not too large to result in saturation effects in the devices. The exponential fit coefficient corresponds to an activation energy of about $11 \mathrm{meV}$, which is consistent with that expected for boron levels in germanium.
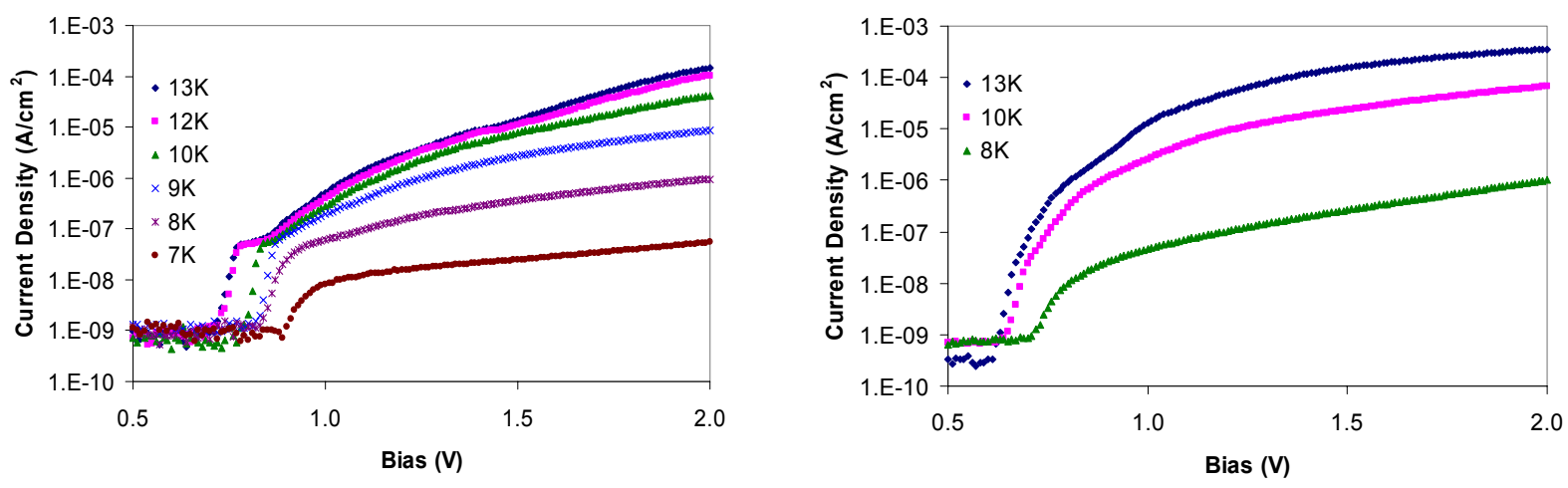

Figure 3. Dark current density vs bias for the smaller germanium detectors (left) and the larger detectors (right). An applied voltage of about $0.7 \mathrm{~V}$ is needed for current to flow, suggestive of non-ohmic contacts 


\subsection{Germanium detector photocurrent characteristics}

Photocurrent was obtained for the larger and smaller detector sizes by applying broad-band, uncalibrated illumination from a blackbody source, filtered through the $75-\mu \mathrm{m}$ cut-on filter, into the test dewar's optical entrance window. Optical performance was better for the smaller area germanium detectors than for the larger ones, as indicated in Figure 5, where representative photocurrent density is plotted vs bias. The smaller detectors turned on more quickly with bias and had a flatter I-V curve after turn-on. The larger detectors turned on more slowly with applied bias and reached only about half the photocurrent density (i.e. QE) as the smaller detectors. This result which is not consistent with normal BIB detector operation was, however, consistent across all detectors in the test package. Given this result, only the smaller detectors were used for subsequent spectral response testing as described below.

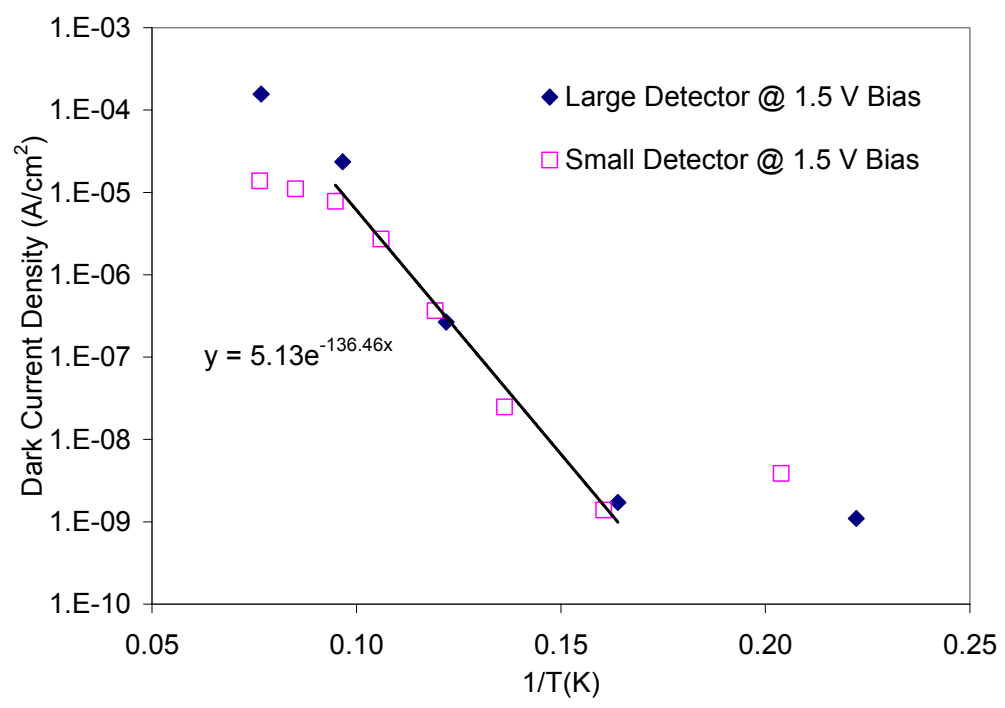

Figure 4. Dark current activation for the germanium detectors

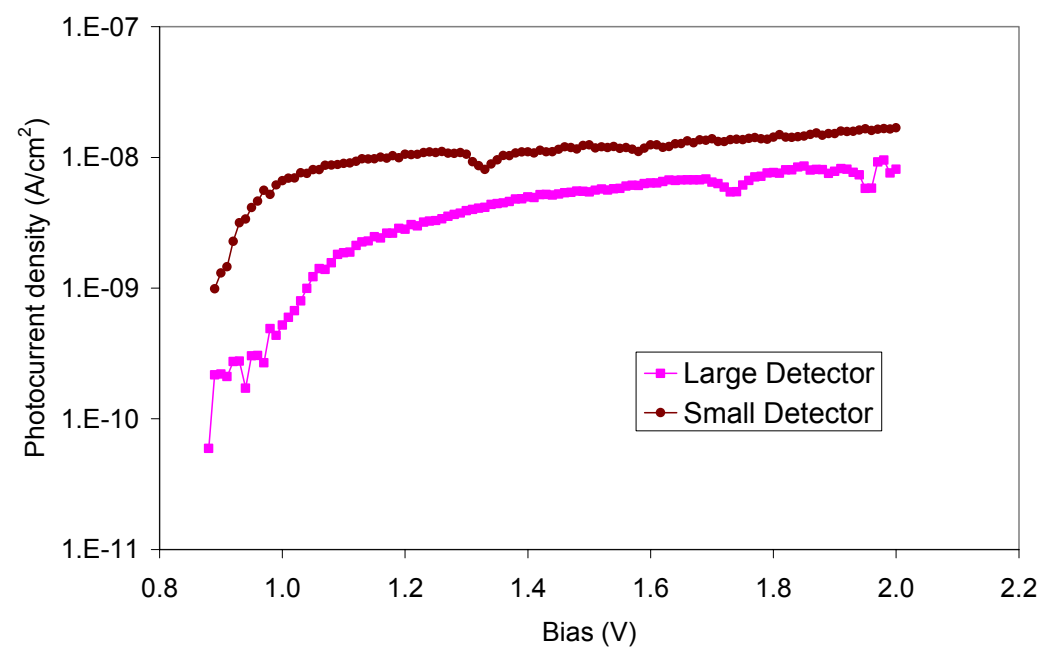

Figure 5. Typical photocurrent vs bias for the larger and smaller germanium detectors 


\subsection{Silicon pilot detector dark performance}

In contrast with the germanium detectors, the silicon detectors, including those of both donor doping levels, operated normally with respect to bias and temperature conditions. There was unusually large variability in dark current among the detectors, but their trends in dark current with size and doping were as expected. Figure 6 plots the dark current density at $4 \mathrm{~K}$ operating temperature vs bias voltage for the 4 detector groups - two doping levels and two detector sizes. The curves are averages over the individual detector curves. The standard deviation of the detector currents was approximately an order of magnitude, which is approximately the difference between the plotted average curves. Thus, the difference between the performance of the smaller and larger detector groups of Figure 6 is not considered significant. The near-exponential functional form of the dark current vs bias curves is evidence of normal BIB detector dark current generation.

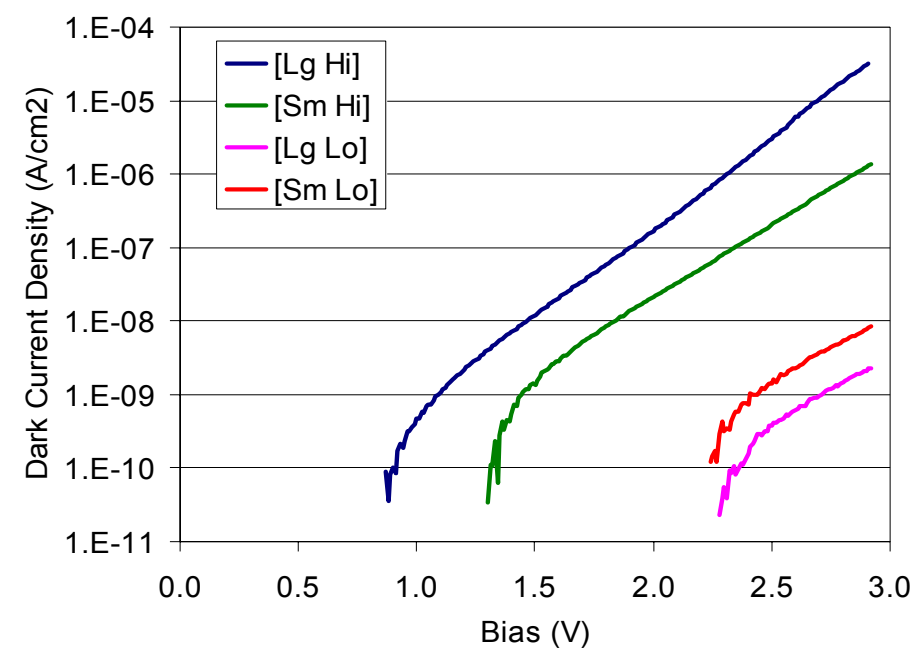

Figure 6. Average dark current density at $4 \mathrm{~K}$ for the four silicon detector groups

To characterize the strong dependence of the BIB detector dark current on doping level at these relatively high doping levels, we compared the temperature dependence of the dark current for a representative small detector of each of the higher (detector S15) and the lower (detector S44) doping groups. A 2-V operating bias was used for each detector, and operating temperature was varied from about $5 \mathrm{~K}$ to about $11 \mathrm{~K}$ in approximate $1 \mathrm{~K}$ steps. Figure 7 plots the dark current vs the reciprocal of the operating temperature, so that trend line representative of thermal activation can be fit to the data. For detector S15 the dark current activation was approximately as expected. The lower-doped detector S44 appeared to become current limited at high temperature. This is probably due to the method used to access the buried contact used in the silicon when they were processed with the existing modified mask set. Variably resistive back contact connections probably account for the significant variability in dark current for the detectors fabricated in this lot. This problem will not occur for the new mask set to be prepared for processing subsequent detectors intended to meet the requirements of Table I. The new detector lot will provide the standard, highly-reliable method for buried contact access, and thus avoid the potential for high-resistance electrical contacts. Taking the lower current region in Figure 7 to provide a valid current comparison, we estimate that approximately doubling the donor doping has lead to a dark current increase of only an order of magnitude at the increased doping levels investigated in the pilot lot. For comparison BIB detectors doped at $10^{18} \mathrm{~cm}^{-3}$ will have at least 4 orders of magnitude greater dark current than corresponding detectors doped at half that level. This suggests that the rate of dark current growth with doping is decreasing for BIB detectors, such that the dark current attending very high doping (to achieve wavelength extension in BIB detectors) may be manageable.

\subsection{Spectral testing of germanium and silicon detectors}

Spectral testing was performed using the set of long-pass infrared filters procured from Lakeshore. Cut-on wavelengths of 30,50,70, and 114 microns were included in the filter set. The filters were used by placing them between a blackbody source and the test dewar's silicon entrance window. By processing the resulting illuminated I-V data in pairs 
corresponding to filtering the blackbody through different cut-on filters, and utilizing knowledge of the black body emission and dewar optical elements, it was possible to estimate the average quantum efficiency of the detectors for the wavelength range corresponding to the difference between the filter cut-on wavelengths. Figure 8 plots the result of that analysis for the germanium detector group and the higher doped silicon detector group.

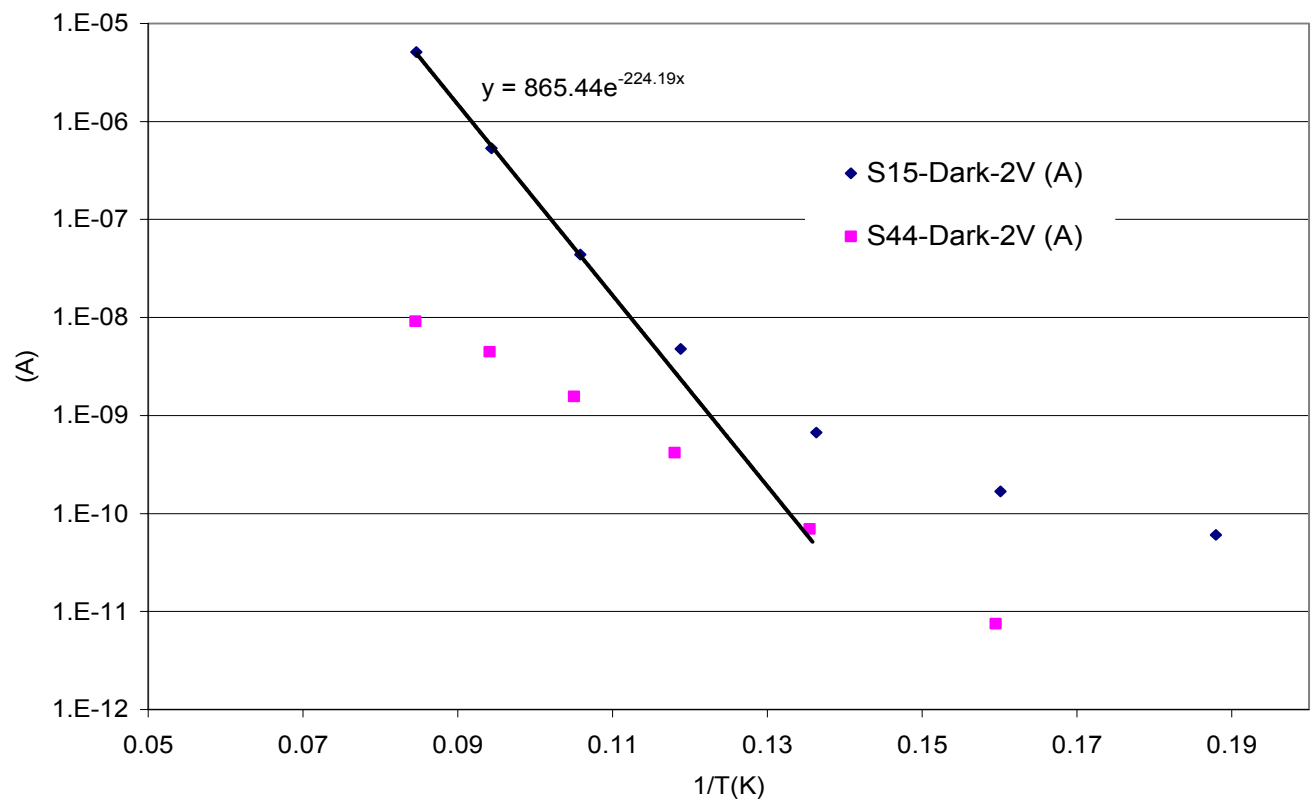

Figure 7. Temperature dependence of the dark current for a representative small ( $0.5 \mathrm{~mm}$ diameter $)$ detector, S15 of higher doping and a small detector (S44) of lower doping

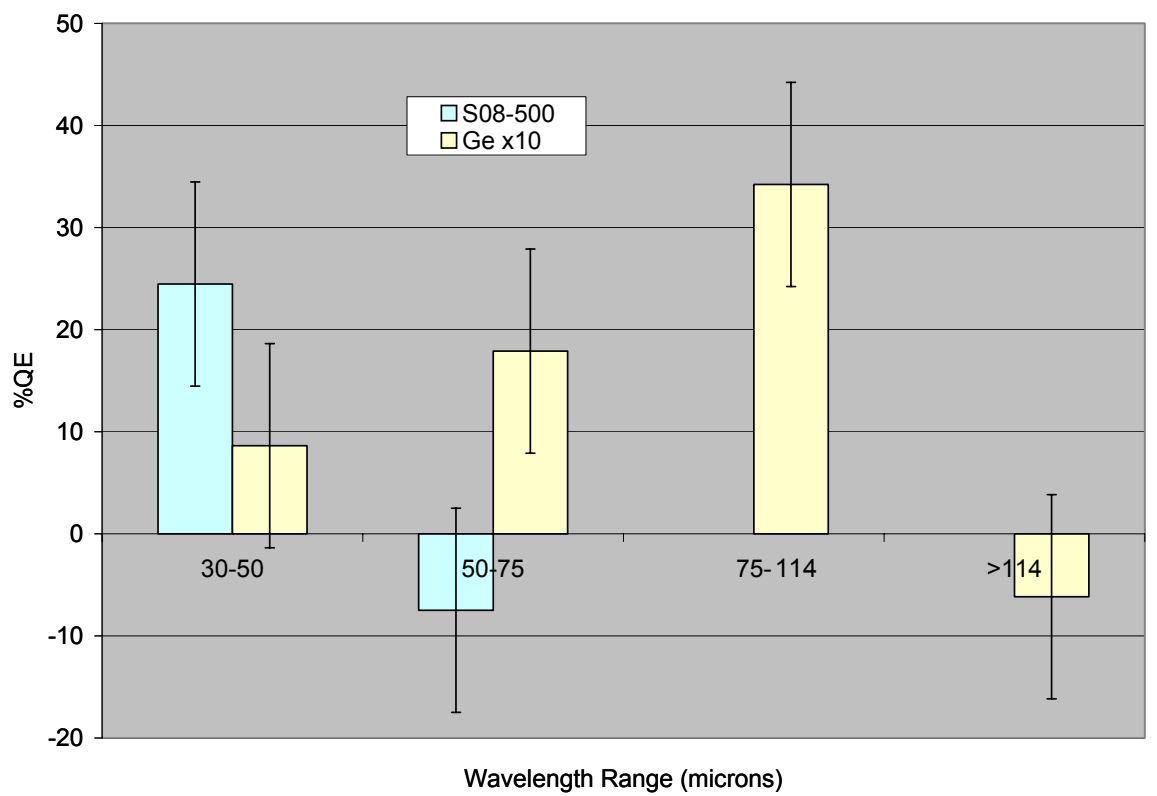

Figure 8. Spectral QE estimated using the differential response of detectors illuminated sequentially through two filters with different cut-on thresholds 
The pilot germanium detectors appear to have spectral sensitivity to wavelengths consistent with the shallow dopant energy levels in that material. However, their operation in opposite polarity or the impurity of the blocking layer leads to their having very small quantum efficiency, only about 3\% at peak. The silicon detectors doped to the highest level available in the pilot detector group, appear to provide high $\mathrm{QE}$ and a degree of wavelength extension well beyond the cut-off for standard Si:As material at 28 microns. However, the direct measurement has poor accuracy (as reflected in the error bars of Figure 8) due to cut-on filters having diffuse cut-on "edges" and a small sub-threshold leakage that has to be subtracted. This inaccuracy does not allow for a quantitative estimate of the wavelength cut-off value to which the higher doped silicon detector wavelength may have been extended by increasing doping level.

In an attempt to estimate the probable cut-off wavelength for the extended wavelength silicon BIB detectors, we utilized the data for the set of filter measurements in a different way. The filter transmission data were fit to a wavelengthextended BIB detector model as shown in Figure 9. The model assumes that the BIB detector QE will remain at approximately at a theoretical value of 50\% (for an uncoated detector) out to a given wavelength where it rolls off at about the same rate with wavelength as does a standard BIB detector. The data from the multiple filter measurements were consistent with a model response that has an effective cut-off between about 50 and 65 microns.

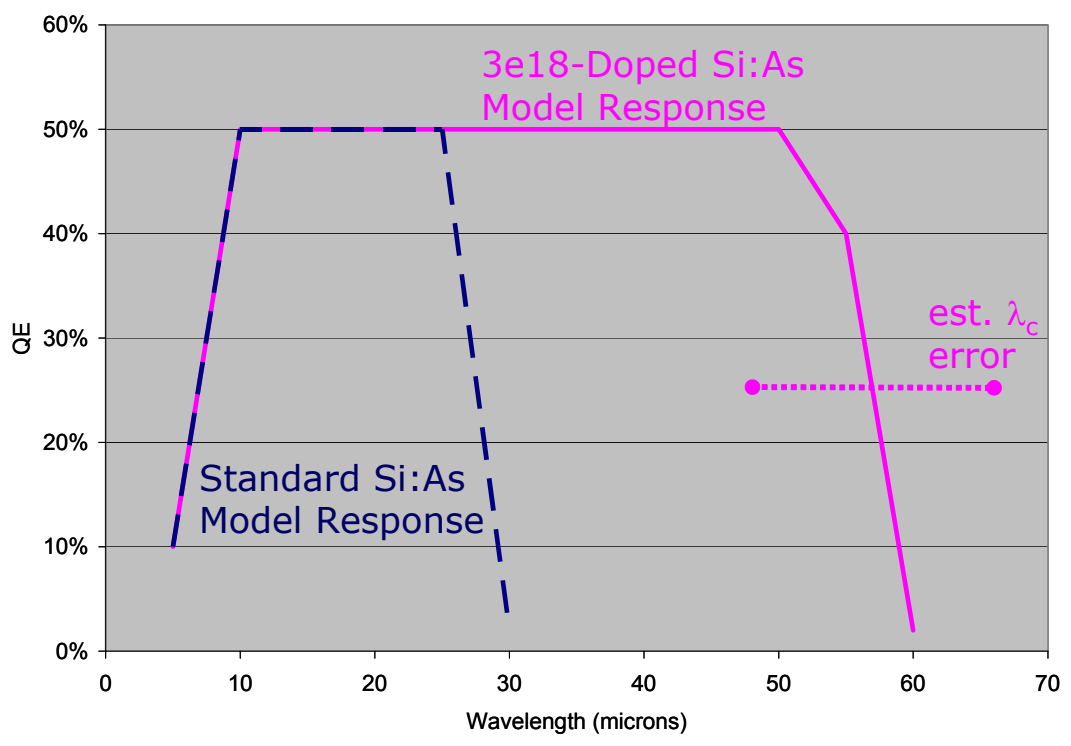

Figure 9. Model extended-wavelength BIB detector response that best agrees with the data taken with the set of cut-on filters

\section{SUMMARY OF PILOT DETECTOR RESULTS}

The attempt to demonstrate extended wavelength BIB detectors by two approaches has met with some initial success with the pilot lot of detectors fabricated from non-optimal available starting materials. The pilot germanium detectors performed anomalously, requiring operation voltage opposite of that expected in order to produce measurable dark and photo response. The characteristic of the germanium dark current was consistent with BIB detector operation; however, the quantum efficiency was very low. The anomalous operation of the germanium detectors is probably due to the impurity levels in the blocking layer, which is reported to have prevented others from achieving effective germanium BIB detectors to date. While our germanium result suggested a structure and operating mode that might be built upon for future success with this detector technology, the silicon detector approach appeared more promising for advancement to the next phase of the development effort.

The Si:As detectors, doped to the highest level available in existing BIB detector material, appeared to have provided detectors that function as generally expected of BIB detectors and to have provided significant optical performance beyond the $28 \mu \mathrm{m}$ cut-off of Si:As doped to standard levels. In a model-dependent estimate the wavelength extension may be as great as a factor of 2 . This was encouraging, since the general performance of the silicon detectors in the pilot 
lot suggested that higher doping levels and consequent longer wavelength extension could be achieved in the next program phase. Based on data from the pilot lot, Table III estimates the performance figures of merit for silicon BIB detectors, assuming their wavelength were extended to 75 microns (the minimum program goal). The detectivity, limited by dark current, exceeds requirements by about 4 orders of magnitude, allowing margin for higher dark current expected to result from higher doping needed to achieve further wavelength extension.

Table III. Anticipated Performance of Extended Wavelength Si:As BIB Detectors

$\begin{aligned} \text { Jdark }(4 \mathrm{~K})= & 5.10 \mathrm{E}-11 \mathrm{~A} / \mathrm{cm}^{\wedge} 2 \\ \text { Jdark }(10 \mathrm{~K})= & 1.61 \mathrm{E}-07 \mathrm{~A} / \mathrm{cm}^{\wedge} 2 \\ \mathrm{~A}= & 4.00 \mathrm{E}-02 \mathrm{~cm}^{\wedge} 2 \\ \text { Noise } / \mathrm{rt} \mathrm{Hz}= & 4.54 \mathrm{E}-14 \mathrm{~A} / \mathrm{rt} \mathrm{Hz} \\ \text { Bandwidth= } & 2.50 \mathrm{E}+03 \mathrm{~Hz} \\ \text { Noise }= & 2.27 \mathrm{E}-12 \mathrm{~A} \\ \text { Wavelength }= & 7.50 \mathrm{E}+01 \mathrm{microns} \\ \text { Responsivity= } & 3.00 \mathrm{E}+01 \mathrm{~A} / \mathrm{W} \\ \text { NEP= } & 7.57 \mathrm{E}-14 \mathrm{~W} \\ \mathrm{D}^{*}= & 1.32 \mathrm{E}+14 \mathrm{~cm} \mathrm{rtHz} / \mathrm{W}\end{aligned}$

\section{INITIATION OF SECOND PHASE DETECTOR DEVELOPMENT}

Silicon BIB detector material parameter selection and material growth for the second phase of detector development have been completed and detector processing has begun. In addition to the continued use of arsenic as a dopant, pushing the concentration to higher levels, we decided to include phosphorus as an alternative dopant. The phorphorus doping chemistry is the same as the arsenic doping chemistry, therefore a Si:P material group could be added for minimal additional cost. The potential advantage of Si:P for wavelength extension is that phosphorus atoms lie at a slightly shallower impurity level than arsenic atoms in the silicon matrix. This provides an initial detector cut-off level (for standard BIB detector dopant concentration) of about $35 \mu \mathrm{m}$ instead of the $28 \mu \mathrm{m}$ of Si:As. It may be relatively easier to extend Si:P detector performance beyond 75 microns. Table 2 lists the detector material selected for the main detector lot processing. The Si:As and Si:P material as $4 \times 10^{18} \mathrm{~cm}^{-3}$ are considered the main candidates for achieving minimum detector performance goals. The higher doping level represents a more aggressive design. Some wafers with standard $\mathrm{Si}: \mathrm{As}$ and $\mathrm{Si}: \mathrm{P}$ levels are included to provide reference detectors for assuring correct lot processing and judging the extent of wavelength extension for the highly doped layer designs.

Table IV. BIB Detector Material for Main Detector Lot

\begin{tabular}{|c|c|c|}
\hline Detector Material & Dopant Concentration $\left.\mathbf{( c m}^{-\mathbf{3}}\right)$ & Number of Wafers \\
\hline Si:As & $1 \times 10^{18}$ & 4 \\
Si:As & $4 \times 10^{18}$ & 4 \\
Si:As & $8 \times 10^{18}$ & 2 \\
Si:P & $7 \times 10^{17}$ & 4 \\
Si:P & $4 \times 10^{18}$ & 4 \\
Si:P & $8 \times 10^{18}$ & 2 \\
\hline
\end{tabular}


DRS and NASA Langley have agreed on the design of the individual detector elements for the new detector lot, and a new detector mask set to be used to fabricate the detectors has been designed and fabricated. The individual detectors will be patterned as $3 \times 3$ arrays of individually accessible square detectors. Detector sizes (side lengths) of 75, 100, 150, and $200 \mu \mathrm{m}$ have been included. Both front- and back-illuminated detector designs have been included on the mask set. These designs allow some flexibility in the material layer structures processed with the mask set. Detectors of this type occupy the central $1 / 3$ of the detector layout on the wafer map. The remainder of the wafer map has been filled with detector array types for which there are existing multiplexed readouts, to allow future demonstration of extended wavelength detector arrays.

\section{ACKNOWLEDGMENT}

The work reported here is sponsored in part by the National Aeronautics and Space Administration Langley Research Center under Contract NNL06AA25C.

\section{REFERENCES}

${ }^{1}$ M G Mlynczak, D G Johnson, M N Abedin, M G Stapelbroek, H H Hogue, and J Reekstin, "The Far-Infrared Detector Technology Advancement Partnership - FIDTAP," in Passive Optical II, Proceedings of the Sixth Annual NASA Earth Science Technology Conference, 2006.

${ }^{2}$ M G Mlynczak, et al, "The far-infrared: A frontier in the remote sensing of Earth's climate and energy balance, in Optical Spectroscopic Techniques, Remote Sensing, and Instrumentation for Atmospheric and Space Research IV, A M Larar and M G Mlynczak, Ed., Proceedings of SPIE, Vol 4485, 191-201, 2001.

${ }^{3}$ M G Mlynczak, D G Johnson, H Latvakoski, K Jucks, M Watson, G Bingham, D P Kratz, W A Traub, S J Wellard, and C R Hyde, "First light from the Far-Infrared Spectroscopy of the Troposphere (FIRST) Instrument," Geophys. Res. Lett., doi: 10.1029/2005GL025114.

${ }^{4}$ M D Petroff and M G Stapelbroek, U S Patent No. 4568960 (4 Feb 1986).

${ }^{5}$ H H Hogue, R B Mattson, M G Stapelbroek, S A Masterjohn, M F Larsen, and J D Elwell, "Focal plane detectors for the WISE 12- and 23- $\mu \mathrm{m}$ Bands" in Infrared Detectors and Focal Plane Arrays IX, Proceedings of the SPIE, Vol. 6660: Infrared Systems and Photoelectronic Technology II, 2007.

${ }^{6}$ A K Mainzer, J Hong, M G Stapelbroek, H H Hogue, D Molyneux, M Ressler, E Atkins, J Reekstin, M Werner, and E Young, "A New Large-Well 1024 x 1024 Si:As Detector for the Mid-Infrared,” Proceedings of the SPIE, Vol. 5881.

7 A G U Perera, H X Yuan, and M H Francombe, "Homojunction Internal Photoemission Far-Infrared Detectors: Photoresponse Performance Analysis," J. Appl. Phys. 77 (2), 15 Jan 1995.

${ }^{8}$ D M Watson, M T Guptill, J E Huffman, T N Krabach, S N Raines, and S Satyapal, "Extrinsic Germanium BlockedImpurity-Band Detector Arrays: Unpassivated Arrays with Bulk Substrates," J. Appl. Phys. 74, 4199. 